\title{
Thermally-induced Spectral Variations of LED applied to Optical Multispectral Camera Communications
}

\author{
Daniel Moreno, Behnaz Majlesein, Julio Rufo, Victor Guerra, Jose Rabadan, Rafael Perez-Jimenez \\ Photonic Technology and Communications \\ IDeTIC \\ Las Palmas de Gran Canaria, Spain \\ dmoreno, bmajlesein, jrufo, vguerra, jrabadan, rperez@idetic.eu
}

\begin{abstract}
Optical Camera Communication (OCC) applications have intensively risen in recent years. This work proposes using a multispectral or hyperspectral camera as an OCC receiver to take advantage of the high spectral resolution of this type of device with respect to conventional cameras. Furthermore, spectral features of the LEDs, usually used as transmitters in OCC, are affected by temperature. Hence, one of the goals of this work is to benefit from those spectral variations that will be grasped by the multispectral camera and thus achieve a new data channel. Therefore, different communication channels could be obtained from a single light source.
\end{abstract}

Index Terms-OCC, multispectral, hyperspectral, LED, temperature dependence, peak wavelength

\section{INTRODUCTION}

$\mathbf{I}$ $\mathrm{N}$ the last few years, there has been a rapid rise in the use of Optical Camera Communication (OCC). It is foreseen that significant advancement in this technology will be implemented increasingly soon thanks to the massive presence of optical cameras in consumer electronic devices such as smartphones, tablets and laptops. What is more, OCC has been added as an extension of IEEE 802.15.7 standard on Visible Light Communications (VLC) [1]. This technology used a camera or an image sensor to receive data from a light source. The sensor scanning is based on two different modes. On the one hand, global shutter exposes every pixel per frame simultaneously. Moreover, it can only detect waveforms with bandwidths below half the frame rate. On the other hand, rolling shutter scans the image sequentially. Besides, this mode allows higher speed communication because the optical source's waveform can be captured within a single frame [2]. Nevertheless, the maximum obtainable speed is dependent on the projected size of the light source, as OCC is based on image-forming optics [3]. OCC has been proposed as a suitable technology in applications such as the Internet of Things (IoT), Vehicular Communication, and Indoor Positioning Systems (IPS). Positioning accuracy of OCC-based IPS is within the centimeter range [4], [5], outperforming any RF-based positioning technique. Vehicle-to-Anything (V2X) is another promising application field of OCC due to the uprising presence of vehicle- mounted optical cameras (generally used for surveillance and security).

Apart from this, regarding the camera used as a receiver in OCC, in this technology has been employed several types of cameras such as Single-Lens Reflex (SLR) cameras [6] or smartphones [7]. In all the cases mentioned above, the devices are used to take pictures and videos within the visible range. Their image sensor only permits the capture of RGB images at most, similar to the human eye. However, multispectral cameras (and hyperspectral cameras) can capture a higher number of communication bands than the RGB bands provided by conventional cameras. These have been used in different sectors such as remote sensing [8], agriculture [9] or biotechnology [10]. In this manner, Optical Multispectral Camera Communication (OMCC) would offer a wide range of opportunities.

Furthermore, the behavior of the Light-Emitting Diodes (LEDs) varies depending on temperature. Some of the characteristics affected are emitted wavelength and luminosity. This work analyzes the LED spectral variations caused by heat. The use of a multispectral (or hyperspectral) camera, which has a high spectral resolution, is proposed to take advantage of this typically considered harmful effect. It is expected to get various independent communication channels using a single sort of transmitter, which would lessen the complexity and cost of the system. On the other hand, although this type of camera has a low sampling rate, there are techniques such as spectral signatures extraction to distinguish particular signals at different wavelengths that would improve its performance.

This paper is divided into six sections. Section II gives a brief overview of the theoretical background on which the work is based. The characterization methodology and the results concerning the temperature effects on the used LED devices are presented in Sections III and IV, respectively. In Section $\mathrm{V}$, the application of this characterization is proposed using a multispectral camera. Lastly, some conclusions are drawn in Section VI.

\section{Temperature DePENDENCE ON LED WAVELENGTH}

The working principle of LED is spontaneous emission of light. That light is emitted when an electron obtains 
enough energy to traverse the forbidden energy gap of the semiconductor. Then, a photon is emitted when that electron goes back to a lower energy level. The emitted photon has a wavelength that is related to the energy gap by Equation 1. In addition, the energy gap is affected by the $\mathrm{p}-\mathrm{n}$ junction temperature, depending on the LED's substrate, which is usually modeled using Equation 2. According to [11], in most semiconductor materials energy gap diminishes as temperature increases. Therefore, as wavelength is inversely proportional to energy gap (Equation 1), the peak wavelength of the LED grows with temperature.

$$
\begin{gathered}
E_{g}=h f=\frac{h c}{\lambda} \\
E_{g}=E_{0}-\frac{\alpha T^{2}}{T+\beta}
\end{gathered}
$$

where $h$ is Planck constant, $f$ is frequency, $c$ is the speed of light in vacuum and $\lambda$ is wavelength. $T$ is temperature, $E_{0}$ is energy gap at $0 \mathrm{~K}$ temperature condition, and $\alpha$ and $\beta$ are semiconductor-dependent constants, which are empirically determined.

However, the metallic content in the LED material must be considered. For instance, [12] proved that the band gap might not follow the decrement as the LED heats up, but it may increase with temperature depending on Indium (In) content in InGaN alloys (green and blue LEDs).

Apart from this, spectral width and luminous efficiency are affected by temperature as well. Normally, the conversion efficiency decreases and the spectral width increases as temperature grows [13]. Based on this, it is intended to use a high spectral resolution camera, such as a multispectral camera, to check if these slight wavelength changes in the LED emission are detectable by it. Then, grasping these generally undesired variations, this work proposes improving communications by increasing the number of communication channels.

\section{Methodology}

The primary goal of this work is to benefit from the spectral variations that temperature effects on LEDs, so that a multispectral camera detects those changes and finally use it for OCC purposes. To accomplish this objective, RGB LEDs have been characterized by focusing on the effect of temperature on their emitted spectrum. Moreover, a multispectral camera has been used to set a communication link with those LEDs as transmitters. Lastly, spectral responses of the emitters have been extracted from the pictures to evaluate the practicability of Optical Multispectral Camera Communications (OMCC) and the use of the LED wavelength variations induced by temperature.

The LEDs under study (common cathode RGB LED) were driven using currents ranging from $10 \mathrm{~mA}$ to 130 $\mathrm{mA}$ by an Ethernet-controlled current source to induce them different junction temperatures. Additionally, the temperature was previously stabilized for 5 minutes. This experimental characterization consisted of three different
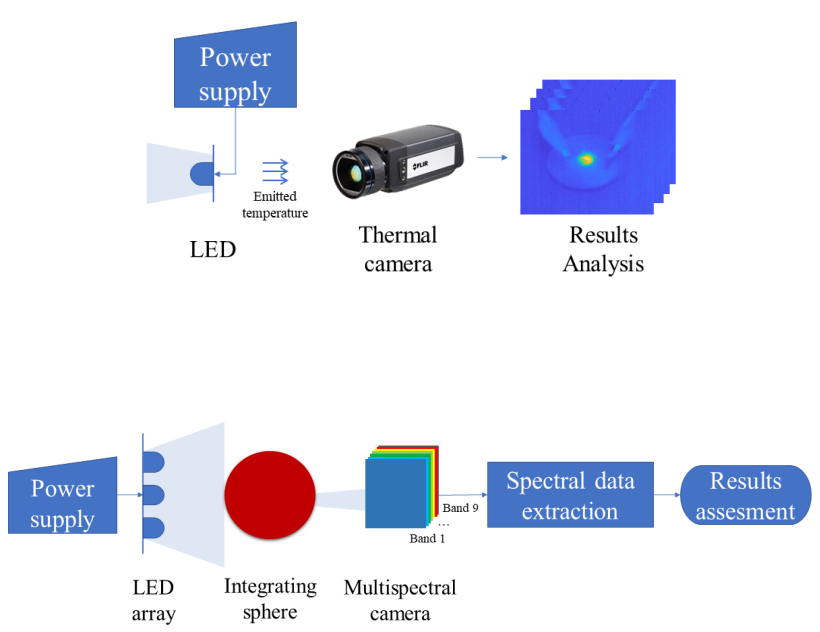

Fig. 1: System setup schemes. Scheme used to get the temperature data (top) and scheme used to get the spectral data (bottom).

processes. Firstly, the junction temperature of the LEDs has been measured by a thermal imaging camera, as shown in Fig. 1 (top). The employed camera was a FLIR A645, whose main features are a pixel resolution of $640 \times 480$ and a high sensitivity below $50 \mathrm{mK}$. For each current, the maximum temperature of the LED has been recorded. Secondly, a spectrometer (Spectral Products SM442) has been used to obtain wavelength data of the LED in the visible range. Due to the high intensity of the LED beam, a 15-centimeters ABS-plastic cylinder has been used to distance the LED from the light input, allowing to receive the signal within the dynamic range of the spectrometer. This fix does not affect the measurement, because of the spectrometer is only needed to get the wavelength information of the LED and visualize shape variations (not absolute changes). Finally, the spectral radiant power of the LED in the visible spectral range has been measured using an integrating sphere and a light meter (Gigahertz BTS256). This device also permits to acquire spectral information; however, the spectrometer provided a better spectral resolution ( $1 \mathrm{~nm}$ vs. $5 \mathrm{~nm}$ ).

After the LEDs' characterization, the aim was to capture the wavelength variation in the LEDs by a camera. It was needed to have a high spectral resolution camera that can detect the slight variations due to temperature; in this case, a multispectral camera, in order to reach this objective. Specifically, the multispectral camera used was the CMSC1-C-EVR1M-GigE by SILIOS Technologies. It is a 9bands camera (8 color narrow bands and 1 panchromatic band) that covers the wavelength range from 400 to 1000 $\mathrm{nm}$. It has a CMOS sensor and a resolution of 1280 (H) $\times 1024(\mathrm{~V})$ in the raw picture and $426(\mathrm{H}) \times 339$ (V) in the spectral pictures. Fig. 1 (bottom) depicts the scheme followed to collect the spectral information of the LED using the multispectral camera. The light beam of these LEDs passes through an integrating sphere that 
concentrates it on a narrow point. Then, the multispectral camera receives that beam and a picture is taken. The camera parameters were adjusted so that the acquired image would not be saturated. The pictures have been saved as single-band images in ENVI file format, which includes one header and one file with multispectral data. Finally, the pictures were cropped selecting only the part that contains the emitted light and from these, the spectral signatures were extracted. With the spectral response of the LED at various temperatures, an evaluation can be made of how well the several signals are distinguished by the camera.

\section{Characterization RESUlts}

In Fig. 2 is presented some examples of the temperaturetime behavior of the RGB LEDs. It depicts an exponential growth which is higher as the driving current increases. Interesting information can be extracted from these graphs, such as that the highest temperature was obtained on the blue LED, followed by the green and the red LEDs. It also can be seen that at $10 \mathrm{~mA}$ the temperature kept almost constant. As an example, Fig. 3 shows several thermal pictures of the red LED at low and high temperatures. Fig. 4 (top) shows the effects of the induced high temperature on the LED emission, by increasing the polarization current, on the peak wavelengths of each RGB color. Table I details the spectral information in Fig. 4 (top) showing the peak wavelengths and the Full Width at Half Maximum (FWHM) values of each spectrum. It can be seen that by rising the temperature the emitted wavelength grew as expected and, consequently, the peak wavelength shifted. The largest shift was obtained in the red LED with a difference of $21.1 \mathrm{~nm}$ between the minimum and the maximum temperature (peak wavelength at $659.6 \mathrm{~nm}$ at $27^{\circ} \mathrm{C}$ and $680.7 \mathrm{~nm}$ at $65^{\circ} \mathrm{C}$ ). It was followed by a 13.5 $\mathrm{nm}$ shift in blue (from $486.1 \mathrm{~nm}$ at $28^{\circ} \mathrm{C}$ to $499.6 \mathrm{~nm}$ at $78^{\circ} \mathrm{C}$ ). In green, the peak wavelength did not increase with temperature in the same way that red and blue did. In this case, the highest peak wavelength was found at the minimum temperature $\left(547.2 \mathrm{~nm}, 27^{\circ} \mathrm{C}\right)$. The green source color of the employed LED is made with InGaN on Silicon ( $\mathrm{Si}$ ). As seen in Section II, In content in this alloy can affect in such a way that when the temperature increases the energy gap also grows, so wavelength decreases. In addition to the peak wavelength shift, it is noteworthy how the width changed with the temperature variation. The most striking change was acquired in blue, having a difference between the width at $28^{\circ} \mathrm{C}$ and $66^{\circ} \mathrm{C}$ of roughly $30 \mathrm{~nm}$. Note that the intensity values were normalized in Fig. 4 (top) because the approach was to analyze only the spectral information.

Fig. 4 (bottom) reports the spectral radiant power of the LED in $\mathrm{W} / \mathrm{nm}$. The highest levels were obtained for the blue LED, followed by green and red. It can be noted that at the maximum temperature, when the LED is supplied with $130 \mathrm{~mA}$, the power decreased with respect to the second maximum. As a consequence of that, temperature expectedly affected the efficiency of the LED.
Furthermore, it shows the wavelength variations, as well as the FWHM changes on temperatures.
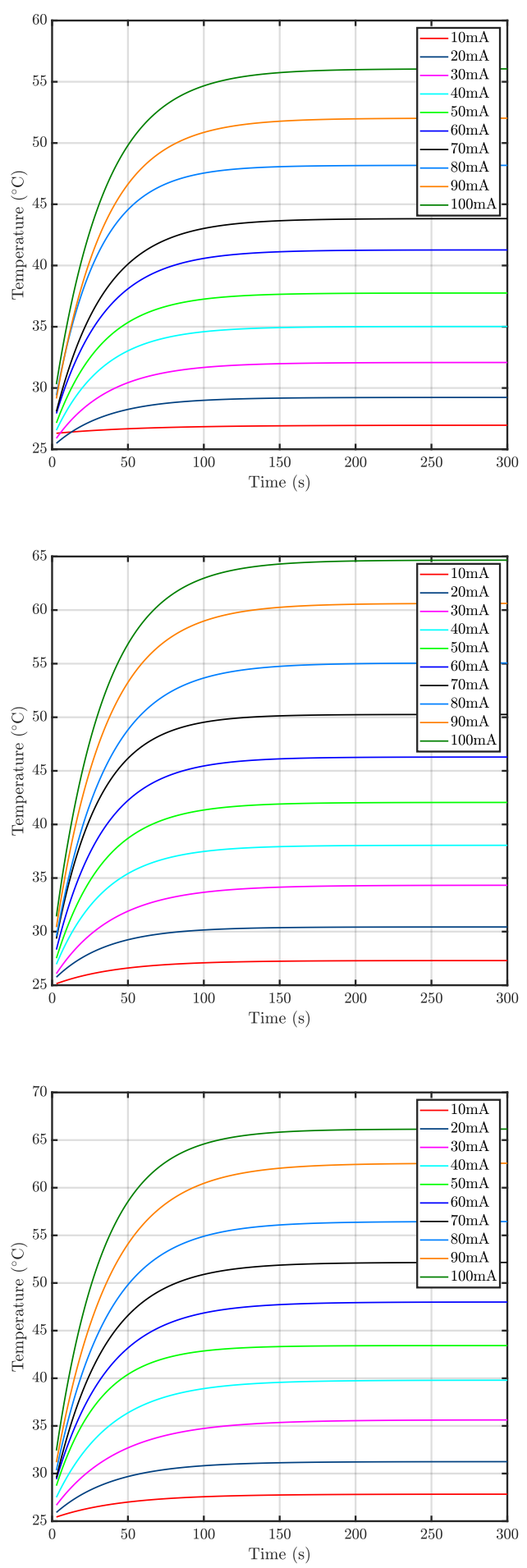

Fig. 2: Temperature behavior of the RGB LED: Red (top), green (middle) and blue (bottom).

\section{Multispectral CAMERA RESUlts}

The images captured by the camera show the spectral variations caused by changing the LED junction temperature. Those changes can be observed in the spectral 

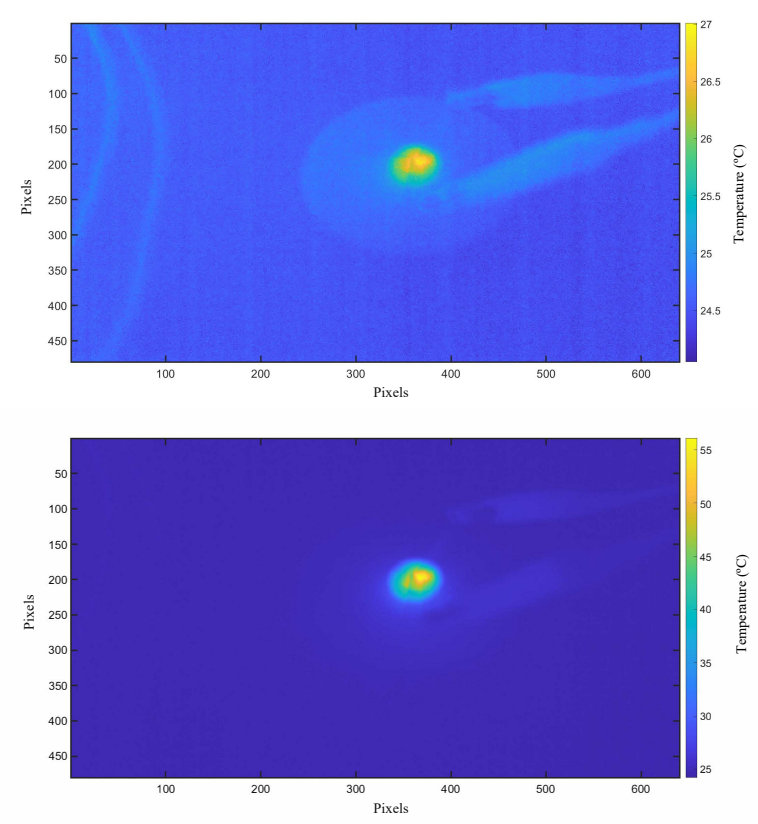

Fig. 3: Examples of images of the red LED driven at different currents, taken by a thermographic camera. LED at $10 \mathrm{~mA}$ (top) and LED at 100 $\mathrm{mA}$ (bottom).

TABLE I: Spectral information at different temperatures.

\begin{tabular}{|c|c|c|c|c|}
\hline LED & \multicolumn{4}{|c|}{ Red } \\
\hline Current (mA) & $10\left(27^{\circ} \mathrm{C}\right)$ & $50\left(38^{\circ} \mathrm{C}\right)$ & $100\left(56^{\circ} \mathrm{C}\right)$ & $130\left(65^{\circ} \mathrm{C}\right)$ \\
\hline Peak wavelength $(\mathrm{nm})$ & 659.6 & 663.6 & 672.5 & 680.7 \\
\hline FWHM (nm) & 8.73 & 14.97 & 22.33 & 24.15 \\
\hline LED & \multicolumn{4}{|c|}{ Green } \\
\hline Current $(\mathrm{mA})$ & $10\left(27^{\circ} \mathrm{C}\right)$ & $50\left(42^{\circ} \mathrm{C}\right)$ & $100\left(65^{\circ} \mathrm{C}\right)$ & $130\left(76^{\circ} \mathrm{C}\right)$ \\
\hline Peak wavelength $(\mathrm{nm})$ & 547.2 & 544.9 & 545.1 & 546.8 \\
\hline FWHM (nm) & 23.73 & 31.80 & 40.92 & 43.18 \\
\hline
\end{tabular}

\begin{tabular}{|c|c|c|c|c|}
\hline LED & \multicolumn{4}{|c|}{ Blue } \\
\hline Current $(\mathrm{mA})$ & $10\left(28^{\circ} \mathrm{C}\right)$ & $50\left(43^{\circ} \mathrm{C}\right)$ & $100\left(66^{\circ} \mathrm{C}\right)$ & $130\left(78^{\circ} \mathrm{C}\right)$ \\
\hline Peak wavelength (nm) & 486.1 & 486.1 & 490.5 & 499.6 \\
\hline FWHM (nm) & 16.68 & 29.62 & 45.85 & 32.10 \\
\hline
\end{tabular}

signatures of the LED shown in Fig. 6. As a case in point, Fig. 6 (bottom) depicts the several levels of energy of the blue LED, supplied with various currents (therefore, at different temperatures), with respect to the 9 bands of the multispectral camera. Targeting the bands 1,2 and 3 , centered in 427,461 and $501 \mathrm{~nm}$, respectively, as the analyzed results were obtained on the blue LED, there are some phenomena to consider. Besides, a high level is placed in band 9, which corresponds to the panchromatic band. However, the focus is not in this band, because the only remarkable consequence is the increase in level due to the increment in current. Firstly, part of the energy captured in the band 1 was captured in the band 2 as temperature grew, causing an increment in the level of this band. The identical happened with bands 2 and 3 . It occurred because the peak wavelength of the LED gradually shifted, so the bands 2 and 3, which have an FWHM of the order of $50 \mathrm{~nm}$ (see Fig. 5), took more information. Additionally, it is perceptible a growth in level as expected, since the way chosen to raise the temperature is by increasing the driving current, ergo the power of the LED also increases. Figs. 6 (top) and 6
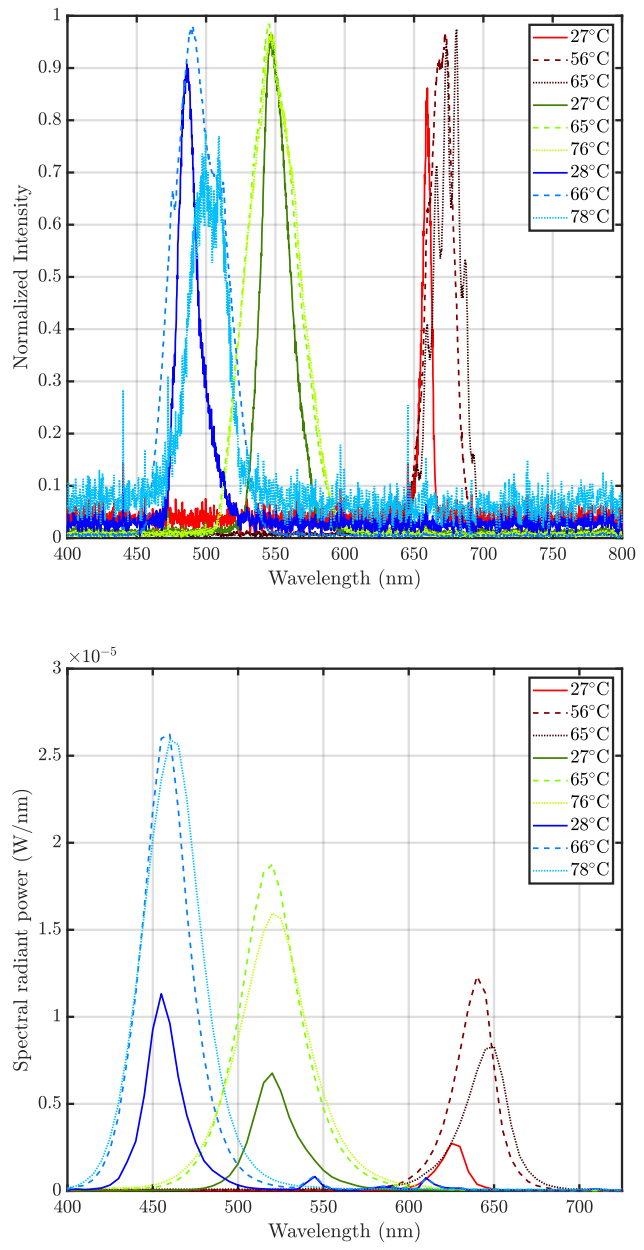

Fig. 4: Characterization of the RGB LED at different temperatures: Normalized RGB LED spectra (top) and spectral radiant power (bottom). Solid line, dashed line, dotted line and dash-dot line correspond to 10 $\mathrm{mA}, 50 \mathrm{~mA}, 100 \mathrm{~mA}$ and $130 \mathrm{~mA}$, respectively.

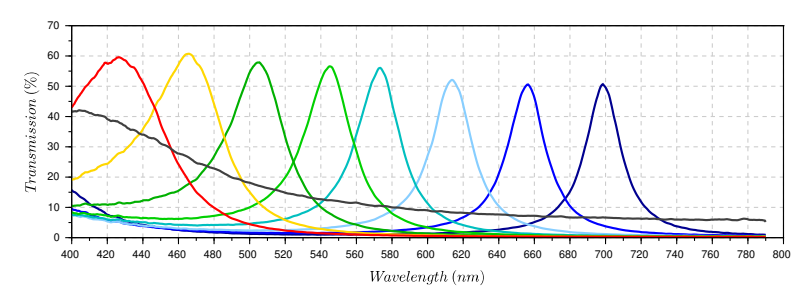

Fig. 5: Filter specifications.

(bottom) depict the spectral signatures of the red and green LED, respectively, showing similar results. These results suggested that several communication channels can be obtained from a single emission source since the slight changes in wavelength are detected by the multispectral camera.

\section{CONCLUSIONS}

In this work, several RGB LEDs have been characterized, especially to visualize the effect of the temperature in the wavelength of the emitted light. Furthermore, it has 

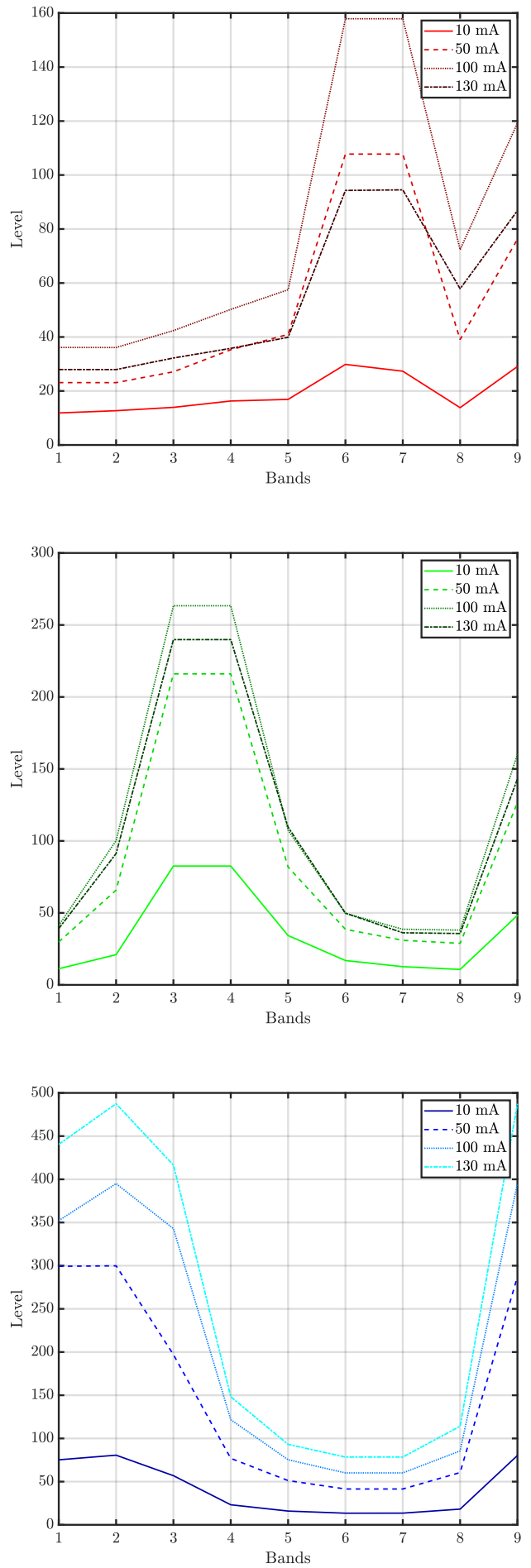

Fig. 6: Spectral signatures of the RGB LED at different temperatures: Red (top), green (middle) and blue (bottom). Solid line, dashed line, dotted line and dash-dot line correspond to $10 \mathrm{~mA}, 50 \mathrm{~mA}, 100 \mathrm{~mA}$ and $130 \mathrm{~mA}$, respectively.

been utilized a multispectral camera taking pictures of the light beam of the LED and analyzing those wavelength variations. The spectral response of the RGB LEDs has been extracted and how the wavelength shifts have been checked. Therefore, as the spectral changes in the emitted light can be observed by using a multispectral camera, it

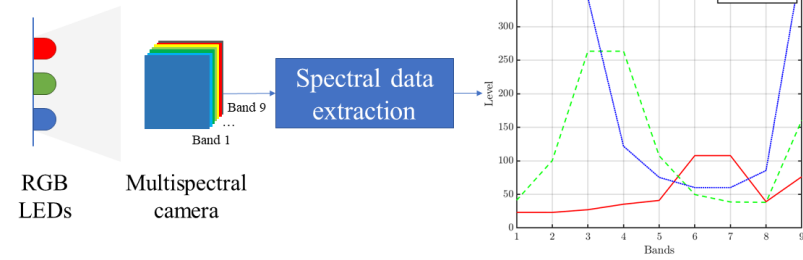

Fig. 7: This figure is an example of a proposed diagram for WDM. By sending three different color signals that are captured by the multispectral camera, after extracting their spectral signatures, each signal could be separated. The same could be performed sending signals of the same color but at different temperatures that shift their spectra and are grasped by the camera.

takes advantage of that phenomenon considered harmful. So, the more variation in the peak wavelength of the LED is affected by temperature, the better. Thus, this paper proposes to use those dissimilarities to create separable communication channels in an OMCC application.

This led us to conclude that employing, for instance, two receivers with two different optical filters, will be feasible to separate each signal by using the same transmitter device. Using the temperature to tune the wavelength of the LED will allow this to be done. It will also be possible to use a camera with enough spectral resolution, such as a multispectral or a hyperspectral camera, which captures the light beam in its several bands with greater or lesser intensity depending on the wavelength shift.

For communication purposes, it would be necessary to do a previous characterization of the transmitters to know how their behavior is affected by the temperature. In this case, the results of this study indicated that the red LED got more considerable spectral variations than green and blue. Moreover, the evidence of this work points towards the idea that temperature effects in emitters, usually considered detrimental, can be detected by highspectral-resolution cameras and used for applications such as Wavelength Division Multiplexing (WDM) (Fig. 7).

Further studies will focus on the use of different transmitters to compare their performance. Additionally, future work will concentrate on creating a channel matrix in order to numerically demonstrate that those signals from a unique transmitter can be discriminated in separable channels.

\section{ACKNOWLEDGMENT}

This work was benefited from the Trainee Predoctoral Research Staff Program (2019) of the Universidad de Las Palmas de Gran Canaria. It has been funded in part by the Canary Islands Regional Government (Project ATICCuA ProID2017010053) and the Spanish Research Administration (MICINN project: OSCAR, ref.: TEC 2017-84065C3-1-R). It has been funded in part by ENLIGHT'EM European Training Network in Low-Energy Visible Light IoT Systems( Innovative Training Networks (ITN) H2020MSCA-ITN-2018 grant number 814215). 


\section{REFERENCES}

[1] Short-Range Optical Wireless Communications (802.15.7-2018), IEEE Revision of the 802.15.7-2011, Rev. 2018, April 2019.

[2] Z. Wang, Q. Wang, W. Huang, and Z. Xu, Visible light communications: Modulation and signal processing. John Wiley \& Sons, 2017.

[3] C. Jurado-Verdu, V. Matus, J. Rabadan, V. Guerra, and R. Perez-Jimenez, "Correlation-based receiver for optical camera communications," Opt. Express, vol. 27, no. 14, pp. 19150-19155, Jul 2019. [Online]. Available: http://www.opticsexpress.org/abstract.cfm?URI=oe-27-14-19150

[4] Y. Li, Z. Ghassemlooy, X. Tang, B. Lin, and Y. Zhang, "A vlc smartphone camera based indoor positioning system," IEEE Photonics Technology Letters, vol. 30, no. 13, pp. 1171-1174, July 2018.

[5] J. Xu, C. Gong, and Z. Xu, "Experimental indoor visible light positioning systems with centimeter accuracy based on a commercial smartphone camera," IEEE Photonics Journal, vol. 10, no. 6, pp. 1-17, Dec 2018.

[6] P. Luo, M. Zhang, Z. Ghassemlooy, H. Le Minh, H.-M. Tsai, X. Tang, and D. Han, "Experimental demonstration of a 1024-qam optical camera communication system," IEEE photonics technology letters, vol. 28, no. 2, pp. 139-142, 2015.

[7] S. R. Teli, S. Zvanovec, and Z. Ghassemlooy, "The first tests of smartphone camera exposure effect on optical camera communication links," in 2019 15th International Conference on Telecommunications (ConTEL). IEEE, 2019, pp. 1-6.

[8] J. Marcello, D. Rodriguez-Esparragon, and D. Moreno, "Comparison of land cover maps using high resolution multispectral and hyperspectral imagery," in IGARSS 2018-2018 IEEE International Geoscience and Remote Sensing Symposium. IEEE, 2018, pp. 7312-7315.

[9] M. Rosenberger, "Multispectral edge detection algorithms for industrial inspection tasks," in 2014 IEEE International Conference on Imaging Systems and Techniques (IST) Proceedings, Oct 2014, pp. 232-236.

[10] F. Meriaudeau, V. Paquit, N. Walter, J. Price, and K. Tobin, "3d and multispectral imaging for subcutaneous veins detection," in 2009 16th IEEE International Conference on Image Processing (ICIP), Nov 2009, pp. 2857-2860.

[11] Y. P. Varshni, "Temperature dependence of the energy gap in semiconductors," physica, vol. 34, no. 1, pp. 149-154, 1967.

[12] C. Zhao, R. Zhang, B. Liu, D. Fu, H. Chen, M. Li, Z. Xie, X. Xiu, $\mathrm{S}$. Gu, and Y. Zheng, "The temperature dependence of optical properties of ingan alloys," Science China Physics, Mechanics and Astronomy, vol. 55, no. 3, pp. 396-399, 2012.

[13] Jeong Park and C. C. Lee, "An electrical model with junction temperature for light-emitting diodes and the impact on conversion efficiency," IEEE Electron Device Letters, vol. 26, no. 5, pp. 308310, May 2005. 